\title{
Image sampling structure conversion by morphological filters
}

\author{
Soo-Chang Pei* \\ Department of Electrical Engineering, National Tain'an Universitı̣, Taipei, Taiwan, ROC \\ Fei-Chin Chen \\ Department of Electrical Engineering. National Taiwan University of Techmolog!'. Taipei, Taiwam, ROC
}

Received 2 December 1991

\begin{abstract}
An efficient image sampling structure conversion using mathematical morphology is described in this paper. The conversion between quincunx and rectangular structures evidently will play an important role in television image processing and HDTV applications. We propose to use morphological filters in this conversion scheme instead of the conventional linear filters. The advantages of the morphological approach over the linear-filtering approach are its direct geometric interpretation, simplicity, and efficiency in hardware implementation. Some image examples are given to show the effectiveness of this approach.
\end{abstract}

Key' words: Sampling conversion; Rectangular sampling; Quincunx sampling; Morphological filter

\section{Introduction}

In the multidimensional signal processing, the conversion between different periodic sampling structures is an important problem, especially for the conversion between quincunx and rectangular structures in television image processing and HDTV applications. For reconstructing the original picture accurately, we have to use appropriate decimation and interpolation low-pass filters. Conventionally, the existing linear programming methods $[3,6]$ are often used to design such filters by incorporating several time- and frequency-

\footnotetext{
${ }^{*}$ Corresponding author.
}

domain constraints. The procedures of design are very complicated, difficult and time consuming for implementation. Also, one may observe a great deal of complexity and cumputation being indispensable in this linear-filtering approach for the sampling structure conversion. In this paper, wu propose to use morphological filters in this conversion scheme instead of the conventional linear filters. The advantages of the morphological approach over the linear-filiering approach are its direct geometric interpretation, simplicity and efficiency in hardware implementation.

We summarize this paper as follows: Section 2 reviews the mathematical morphology operations on binary images and gray-level images. Section 3 illustrates the image sampling conversion methods 
using morphological filters. Section 4 gives some image examples to show the effectiveness of this approach. The conclusions are finally made in Section 5.

\section{Mathematical morphology}

Mathematical morphology is an approach to the image processing based on set theory concepts of shapes $[4,5]$. An image can be represented by a set of pixels, the morphological operations deal with two images: the original data to be analyzed and a structuring element, which is analogous to the kernel of a convolution operation. Each structuring element has a shape which can be regarded as a parameter to the operation. The four basic operations in mathematical morphology are dilation, crosion, opening and closing. This mathematical field was introduced by Matheron [4]. Serra presents these concepts in detail [5]. A tutorial is found in Haralick et al. [2].

\section{I. Binary image morphology}

We assume $X$ (original image) and $B$ (structuring element) to be the subsets of a two-dimensional (2-D) Euclidean space, and let $(X)_{b}$ denote the translation of $X$ by the vector $h$,

$$
(X)_{h}=\{y \mid y=x+h, x \in X\},
$$

then the two fundamental morphological operations, dilation and crosion, can be defined as follows:

$$
\text { Dilation: } \begin{aligned}
X \oplus B & =\bigcup_{b \in B}(X)_{b} \\
& =\{y \mid y=x+b, x \in X, h \in B\},
\end{aligned}
$$

Erosion: $\quad X \ominus B=\bigcap_{b \in B}(X)_{-b}$

$$
=\{y \mid b \in B \text {, implies }(y+b) \in X\} .
$$

This means that a dilation of $X$ by $B, X \oplus B$, can be described as the union of translations of $X$ by all vectors $h$ contained in the structuring element $B$. For erosion, $X \ominus B$, the result is an intersection of translation by all vectors $-b$, where $b$ is contained

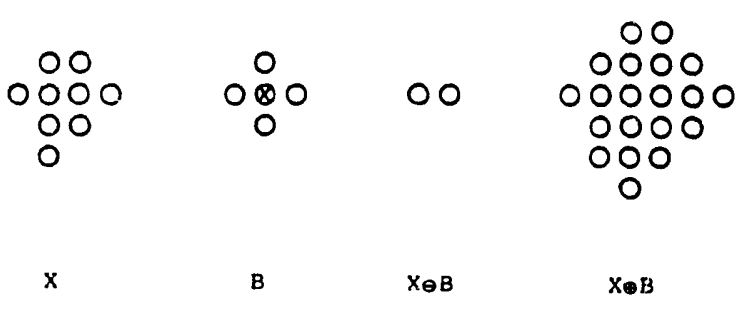

Q denotes the origin of the structuring element B.

(a)

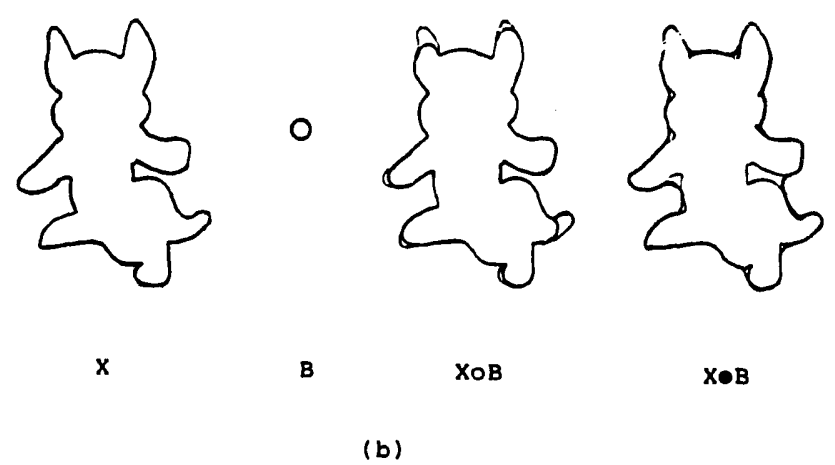

Fig. 1. Example of binary image morphology: (a) erosion and dilation of $X$ by $B$ : (b) opening and closing of $X$ by $B$ (the dark solid curves correspond to the boundaries of the transformed sets).

in $B$. Fig. 1(a) shows the example of erosion and dilation of $X$ by $B$. Dilation and erosion cause the expanding or shrinking of areas when the structuring element has a disk-like shape. Although dilation and erosion are complementary morphological operations, they are noninvertible. This means that the original image, in general, cannot be recovered by performing dilation to its eroded version or vice versa.

An opening is defined as an erosion followed by a dilation by the same structuring element and is shown as

Opening: $\quad X \circ B=(X \ominus B) \oplus B$.

On the other hand, we define a closing as a dilation followed by an erosion as

Closing: $\quad X \bullet B=(X \oplus B) \ominus B$.

Openings on an image with a structuring element $B$ can be pictured by moving $B$ inside all the shapes 
of an image and marking only those places where $B$ fits. Similarly, closings on an image with a structuring element $B$ can be pictured by moving $B$ arsund the outside of all shapes of an image with the result that the concave corners are rounded and the convex corners remain unchanged. The example of opening and closing of $X$ by $B$ is shown in Fig. 1(b).

\subsection{Gray-scale morphology}

Morphological concepts can be extended to gray-scale images. In the gray-scale morphology [2], gray-scale images are visualized as threedimensional (3-D) landscapes with the gray values as third dimension. The structuring elements of the gray-scale morphology are 3-D shapes, like spheres or cylinders. Performing morphological operations on a gray-scale image by a sphericalstructuring element is equivalent to sliding a sphere across the gray-level surface.

Let the image $X(x)$ be represented as a function of coordinates $x$. The analytical definitions of the gray-level morphology operations are as follows:

Dilation: $X \oplus B=D(x)=\max _{b \in B^{\prime}}[X(x-b)+B(b)]$,

Erosion: $X \ominus B=E(x)=\min _{b \in B^{\prime}}[X(x+b)-B(b)]$,

where the $B(b)$ 's are weights that are a function of $b$, and $B^{\prime}$ is the region of support of the structuring element $B$.
Note that all of the relationships for binary in!age morphology are preserved here in a form in which intersection is replaced by min, while union is replaced by max. The opening and closing operations for gray-level images are defined similarly as in the binary case, i.e.

Opening: $\quad X \circ B=(X \ominus B) \oplus B$.

Closing: $X \bullet B=(X \oplus B) \ominus B$,

In gray-level morphology the disk shaped structuring element shown in Fig. 2(a) is 3-D. The result of a 3-D opening is to move the top areas under the top surface of the landscape in Fig. 2(b) defined by the image, and keep those areas where the diskshaped structuring element fits. Strongly peaked areas with widths less than the diameter of the disk-shaped structuring element will flatten out as illustrated in Fig. 2(c).

\section{Sampling structure conversions using morphological filters}

\subsection{Conversion between orthogonal and quincunx structures}

The orthogonal and quincunx periodic sampling structures are the most commonly used in television image processing. The relationship between the $(L, K)$ quincunx lattices in the spatial and frequency domains is shown in Fig. 3, where $T 1$ and $T 2$ represent the sampling period in the horizontal and vertical directions for a spatial sampling pattern. $L$ and $K$ are the parameters of the quincunx sampling structure. A spectral representation of the $(L, K)$ quincunx sampling structure in frequency domain is given in Fig. 3(b). The conversion from

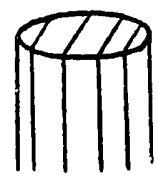

(a)

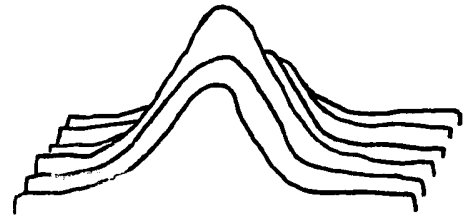

(b)

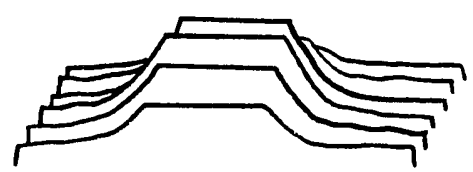

(c)

Fig. 2. Example of gray-level image morphology: (a) disk-shaped structuring element; (b) gray-level image landscape: (c) opening by a disk-shaped structuring element. 


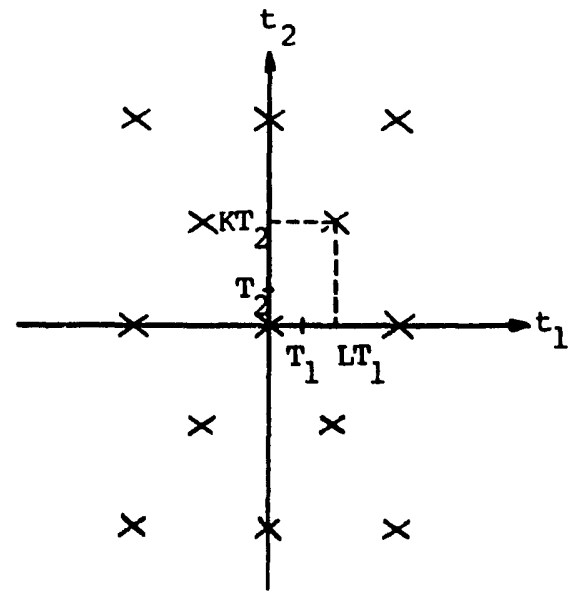

(a)

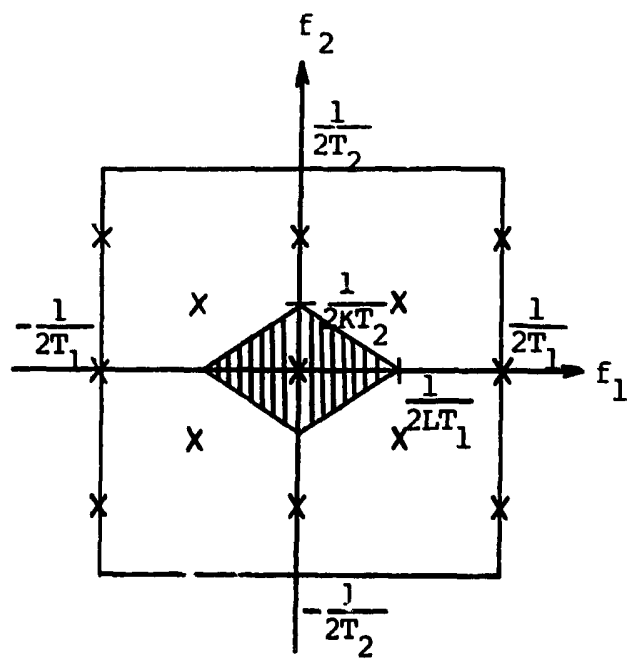

(b)

Fig. 3. $(l, K)$ quincunx sampling: (a) sample points in the $\left(t_{1}, t_{2}\right)$ plane; (b) spectrum centers (denoted by $\left.x\right)$ and the ideal diamondshaped filter $(l .=2 . K=3)$.

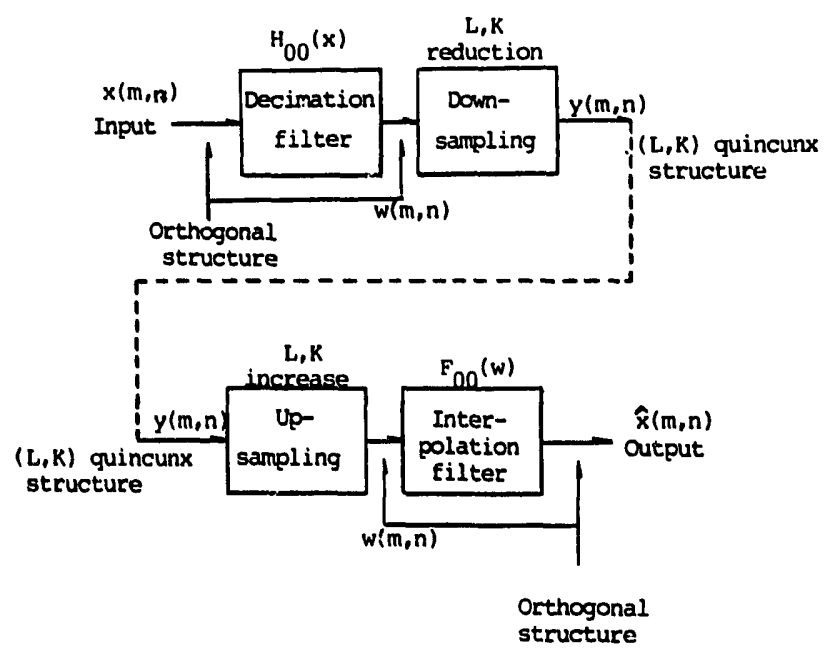

Fig. 4. Block diagram of the sampling structure conversions: decimation interpolation system using 2-D morphological filters.

orthogonal structure to quincunx structure or vice versa corresponds to a decimation or to an interpolation. It is shown that the marked ideal diamondshaped decimation filter or interpolation filter in Fig. 3(b) is good candidate for conversion processing, because it allows a maximum definition in the horizontal and vertical directions. It is well known that human visual perception in the horizontal and vertical directions is better than in the diagonal direction. Siohan [6] and Knoll [3] have recently designed these 2-D diamond-shaped linear FIR filters using complicated optimization techniques which are very time-consuming. The type of symmetry in the ideal diamond-shaped filters will depend on the respective values of the parameters $L$ and $K$, and will be tetragonal $(L \neq K)$ or octogonal $(L=K)$. In consideration of the block diagram of the sampling structure conversions shown in Fig. 4, the orthogonal structure input in Fig. 4 is filtered first through the diamond-shaped 
decimation filter $\boldsymbol{H}_{00}$ and then downsampled to the $(L, K)$ quincunx structure output. For the inverse process, the quincunx structure is unsampled by $(L, K)$ times and then interpolated to become the orthogonal structure output by the diamondshaped interpolation filter $F_{00}$. For the purpose of converience of transmission, the decimation is a first step with the effect of bandwidth reduction, then an interpolation is carried out at the receiver in order to recover the original signal. The natural consequence of this type of processing is a distortion which depends on the values of the conversion parameters, and also on the deviation from the ideal frequency characteristics.

The morphological low-pass filters are used here to substitute the linear decimation/interpolation filters. With mathematical morphology filter technologies, the complexity of the filter design and implementation are greatly reduced on the ground that time-consuming optimization procedures and multiplication operations used in linear filters can be omitted. Image objects are most naturally perceived as geometrical shapes. Therefore, a need exists for representation that emphasizes geometric structures. Mathematical morphology can provide a unique geometric representation of an image by analyzing its shape and conveying its size, orientation, and connectivity. Sequential alternating application of the morphological operations of opening and closing by means of the same structuring element removes details of the image that are small in comparison of this structuring element. We call these alternating sequential filters as morphological low-pass filters:

\section{1-D low-pass decimation filter $\boldsymbol{H}_{0}$ : closing [opening $(X)$ ],}

1-D low-pass interpolation filter $F_{0}$ : closing $(W)$.

The typical quincunx sampling patterns for different $L, K$ parameters are shown in Figs. 5(a)-5(c). Note that only one-half, one-fourth or one-eighth of the image samples are remained in each sampling conversion case. The system diagrams of the sampling structure conversions using morphological filters are shown in Figs. 6 and 7, where the two $3 \times 1$ or $5 \times 1$ decimation structuring

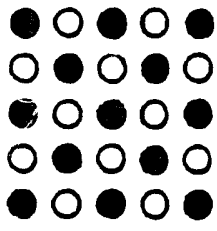

(a)

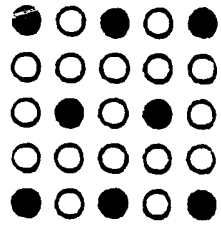

(b)

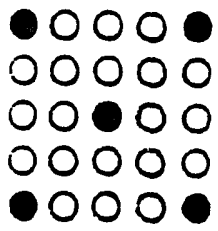

(c)
Fig. 5. Quincunx sampling: (a) $L=K=1$; $\quad$ (b) $L=1, K=2$; (c) $L=K=2$.

elements and $3 \times 2$ or $5 \times 2$ interpolation structuring elements are also shown for reference. The black dots in the $3 \times 3 / 5 \times 5$ windows denote the structuring element, and the gray-scale values of these dots are constant. In analogy to the linear filters one can imagine that the length and direction of the diagonal structuring elements will determine the passband bandwidth and spectrum orientation of the filters. The larger the structuring element is, the narrower the filter passband becomes. The downsampling (upsampling) procedure deletes (interpolates) the image samples on every other diagonal with the inclination angle $\theta=\tan ^{-1}(L / K)$. The 2-D diamond-shaped decimation and interpolation filters are easily designed by a separable product of 1-D $\theta$-degree diagonal and $(\pi-\theta)^{\circ}$ diagonal morphological filters, for example, $\theta=45^{\circ}$ for $L=K=1$ in Fig. 6 and $\theta=27^{\circ}$ for $L=1$, $K=2$ in Fig. 7, i.e. for $\theta=45^{\circ}$ :

2-D low-pass decimation filter $H_{00}$ :

$$
H_{0}^{135}\left[H_{0}^{45}(X)\right]
$$

2-D low-pass interpolation filter $F_{00}$ :

$$
F_{0}^{135}\left[F_{0}^{45}(W)\right]
$$

where $H_{0}^{45}\left(F_{0}^{45}\right)$ and $H_{0}^{135}\left(F_{0}^{135}\right)$ are the $45^{\circ}$ diagonal and $135^{\circ}$ diagona! low-pass filters, respectively.

By means of mathematical morphological filters, the complexity of filter design and implementation is greatly reduced because only summation/subtraction operations and $\max / \mathrm{min}$ decisions are needed. Two opening and closing operations (four dilation and four erosion operations) are used for the morphological filter in Fig. 6(a). In this connection, the computational complexity can be counted as four max and four min decisions per pixel. The summation and subtraction are not needed 


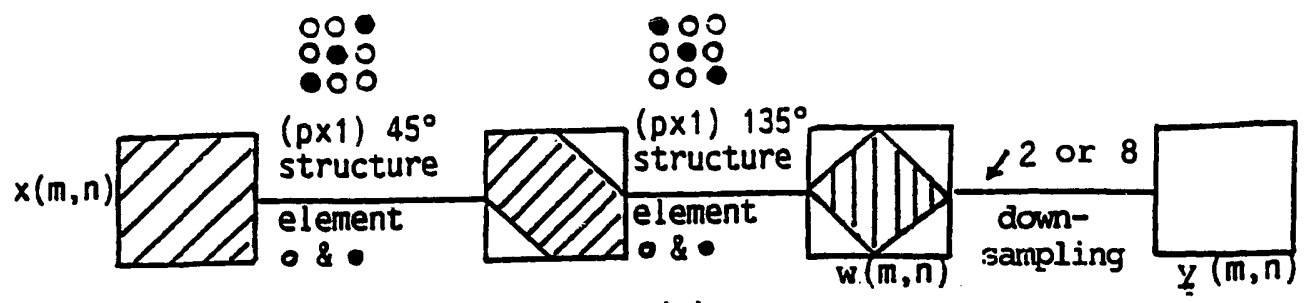

(a)

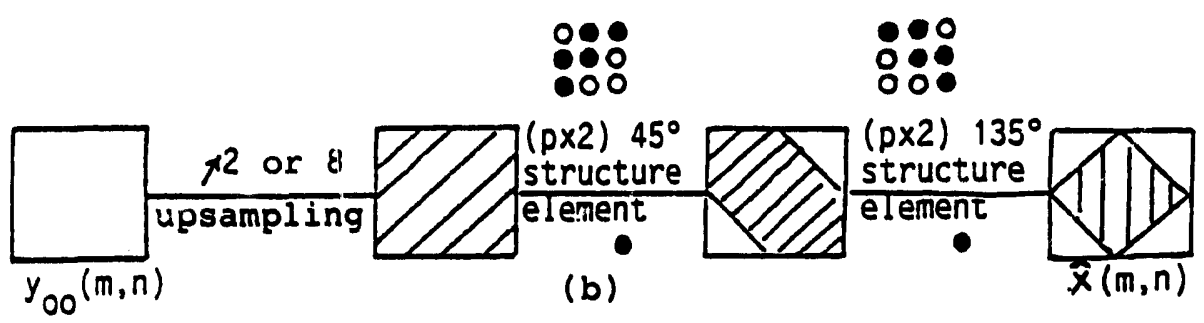

Fig. 6. System diagram of the sampling structure conversions using morphological filters: (a) from the orthogonal to the $(L, K)$ quincunx structure, $L=K=1$ or $L=K=2$; (b) from the $(L, K)$ quincunx to the orthogonal structure.

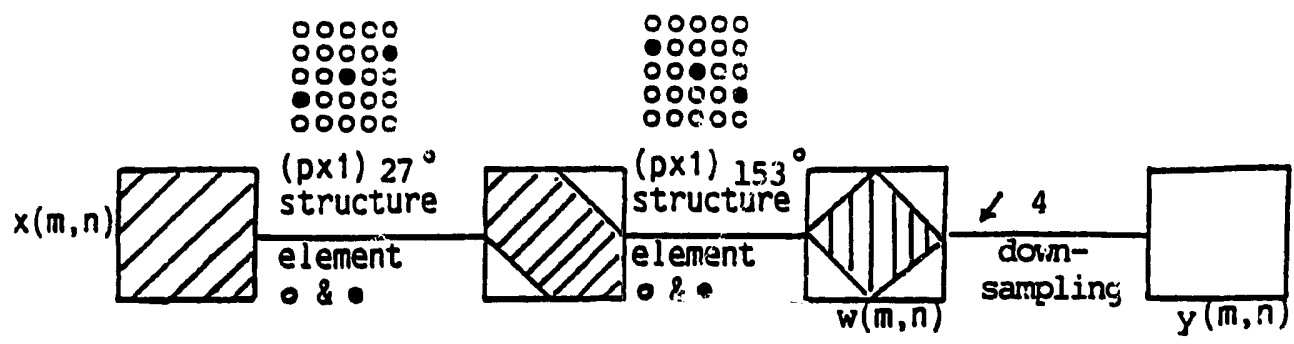

(a)

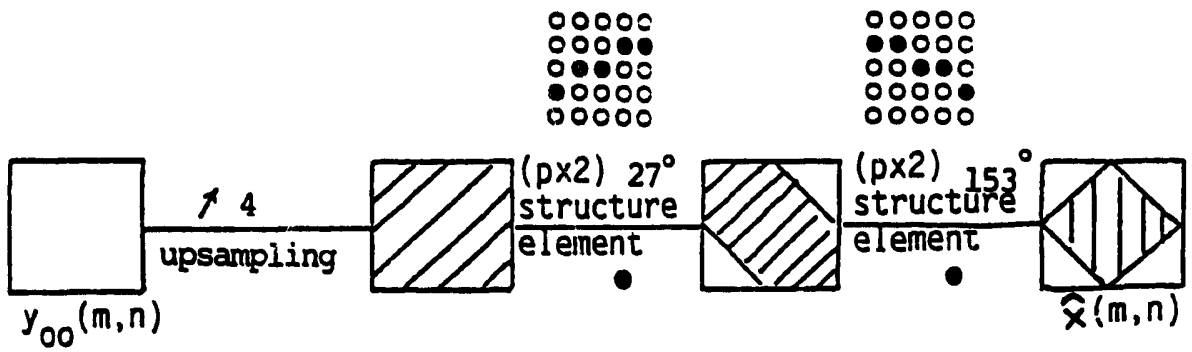

(b)

Fig. 7. System diagram of the sampling structure conversions using morphological filters: (a) from the orthogonal to the $(L, K)$ quincunx structure, $L=1, K=2$; (b) from the $(L, K)$ quincunx to the orthogonal structure.

in dilation and erosion if we give the value of zero to the gray-scale values of the structuring element. On the other hand, if the morphological filter in Fig. 6(a) is replaced by the 2-D $3 \times 3$ linear filter, the computational complexity is nine multiplications and eight summations per pixel. Meanwhile, the processing speed of morphologica! filters is very fast because the basic morphological operations 
can be implemented in the pipeline processing form of parallelism [1].

\subsection{Conversion from the $\left(L_{1}, K_{1}\right)$ quincunx} structure to the $\left(L_{2}, K_{2}\right)$ quincunx structure

To take into account a sampling conversion from an $\left(L_{1}, K_{1}\right)$ to an $\left(L_{2}, K_{2}\right)$ quincunx structure, Fig. 8 shows that cascaded design of the interpolation and decimation processes is sufficient to achieve this conversion process. We denote $F(W)$ and $H(S)$ as the morphological low-pass filters associated with the up- and down-sampling operations, respectively. It can be seen that the two filters $F(W)$ and $H(S)$ operate on the same rectangular (orthogonal) sampling structure. Therefore, the more efficient implementation can be obtained if the two ideal

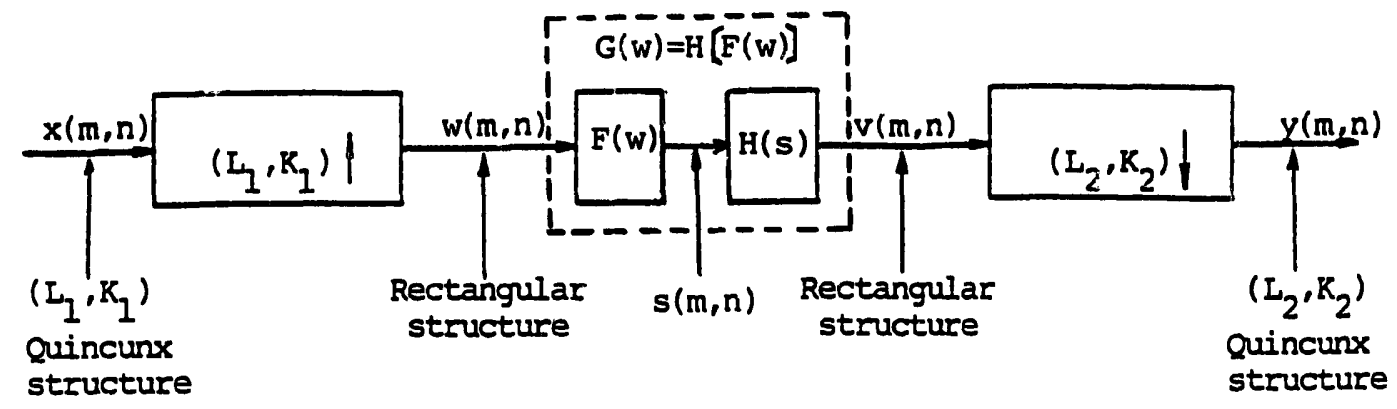

Fig. 8. Conversion from the $\left(L_{1}, K_{1}\right)$ to the $\left(L_{2}, K_{2}\right)$ quincunx structure.

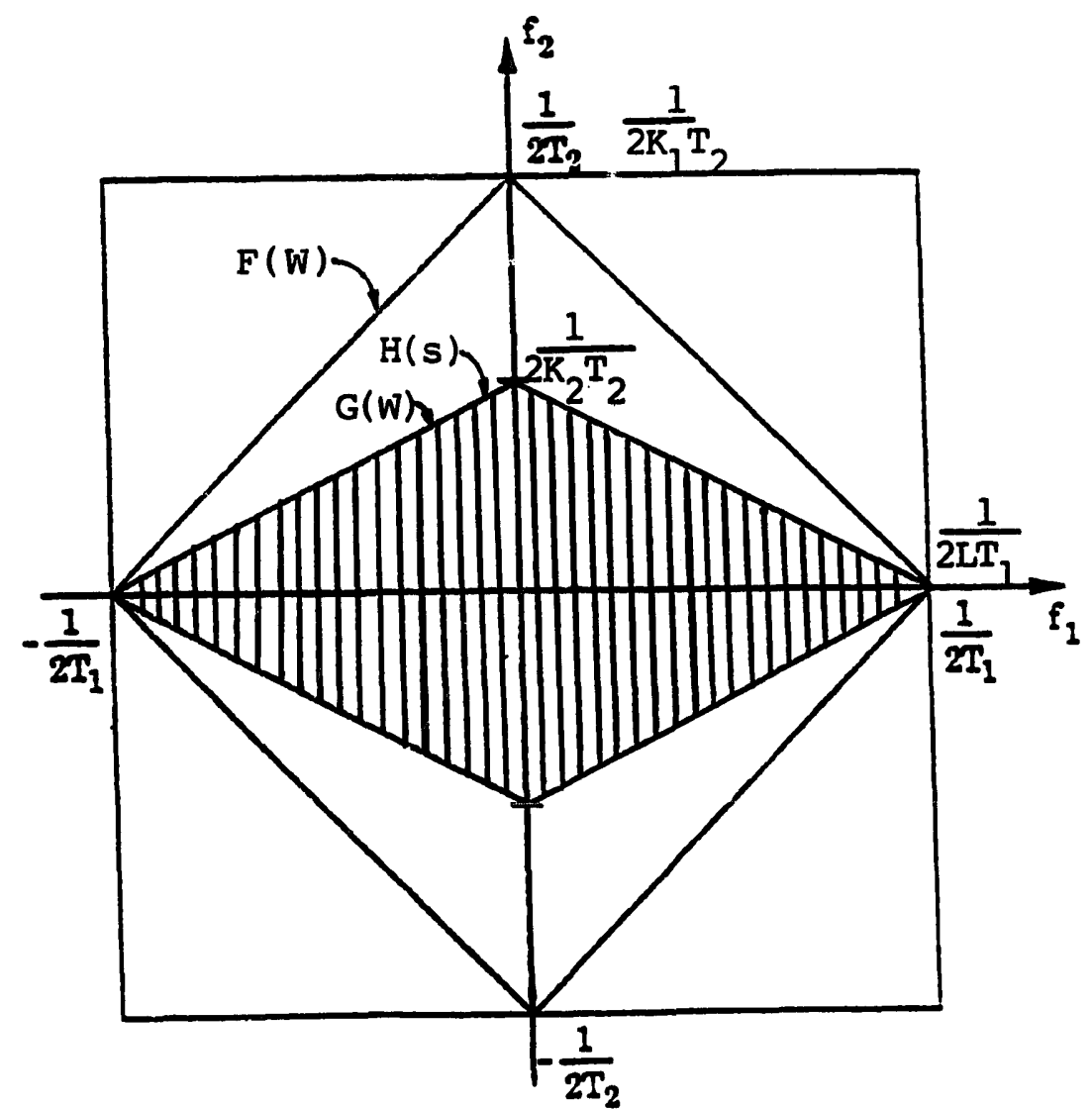

Fig. 9. Contours of the ideal filters for a conversion between two quincunx sampling structures $\left(L=L_{1}=L_{2}=1, K_{1}=1, K_{2}=2\right)$. 


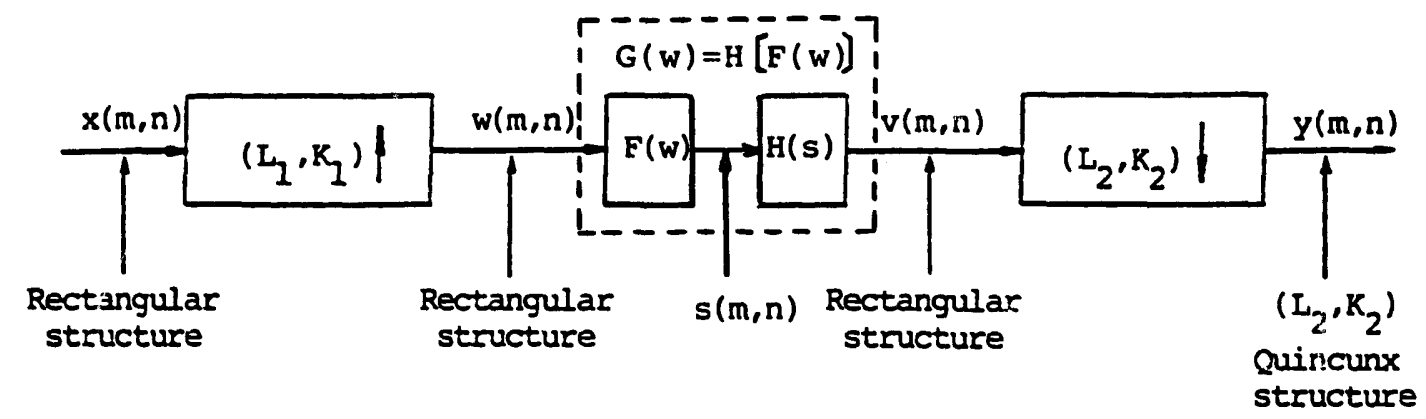

Fig. 10. Conversion from the $\left(L_{1}, K_{1}\right)$ rectangular to the $\left(L_{2}, K_{2}\right)$ quincunx structure.

filters are combined to form one composite morphological low-pass filter, denoted by $G(W)$. The frequency spectrum of the two filters $H(S)$ and $F(W)$ are the small and the big diamond-shaped areas, respectively, as shown in Fig. 9. The common part of these two areas is the frequency spectrum of the filter $G(W)$ and is shown as the shaded area in Fig. 9.

\subsection{Conversion from the $\left(L_{1}, K_{1}\right)$ rectangular structure to the $\left(L_{2}, K_{2}\right)$ quincunx structure}

Assume we want to convert the rectangular structure into a quincunx sampling structure with rational factors $p:=L_{2} / L_{1}$ and $q=K_{2} / K_{1}$. Fig. 10 illustrates a cascade of the interpolation and decimation processes to achieve this sampling conversion. Similarly, the two morphological filters $F(W)$ and $H(S)$ can be combined to form a composite morphological low-pass filter $G(W)$. The frequency spectrum of the two filters $H(S)$ and $F(W)$ are the diamond shaped and rectangular areas, respectively, as shown in Fig. 11. The common part of these two areas is the frequency spectrum of the filter $G(W)$ and is shown as the shaded area in Fig. 11.

\section{Experimental results and discussions}

In this section, some experiments are described to demonstrate the effectiveness of the morphological approach. Figure 4 shows the experimental block diagram. The orthogonal input signal first passes through a decimation filter and down samp-

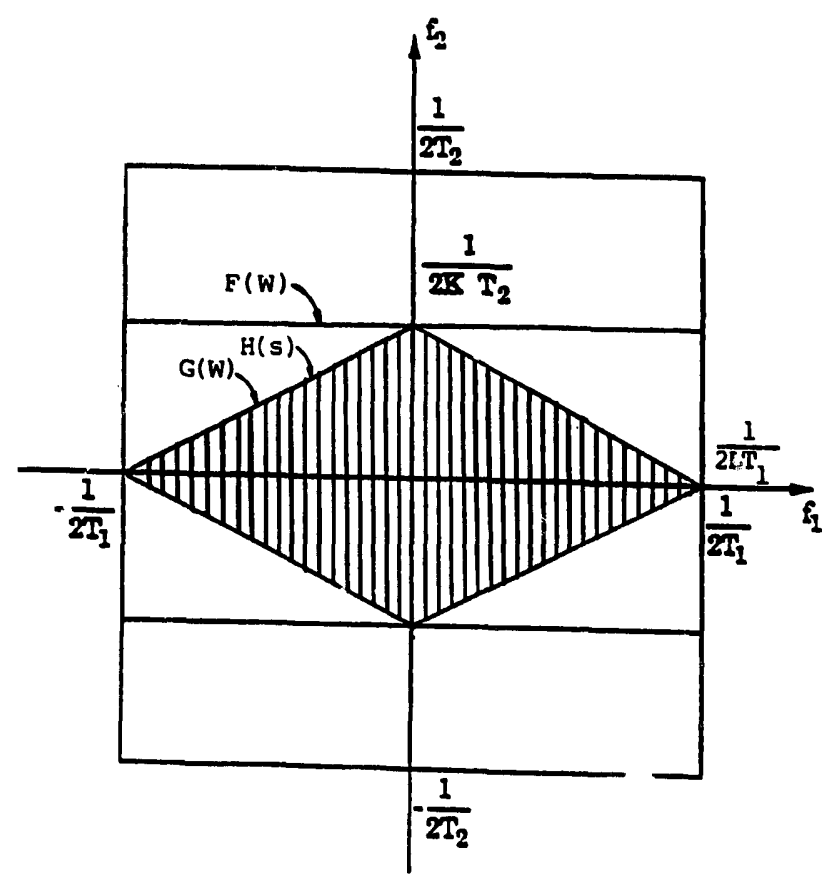

Fig. 11. Contours of the ideal filters for a conversion from the $\left(L_{1}, K_{1}\right)$ rectangular to the $\left.: L_{2}, K_{2}\right)$ quincunx structure $\left(L=L_{1}\right.$ $=L_{2}=1, K=K_{1}=K_{2}=2$ ).

ling operation is performed afterwards. Then the middle quincunx sampled signal is upsampled and passed through an interpolation filter to obtain the final orthogonal output. The distortion and deviation of the cutput from the input can be utilized to evaluate this type of sampling conversion process with the designed 2-D diamond-shaped morphological filters.

The first experiment is processed by using the morphological decimation and interpolation filters with $3 \times 3$ structuring elements for the parameters $L=K=1$. The test picture 'LENA' is shown in 

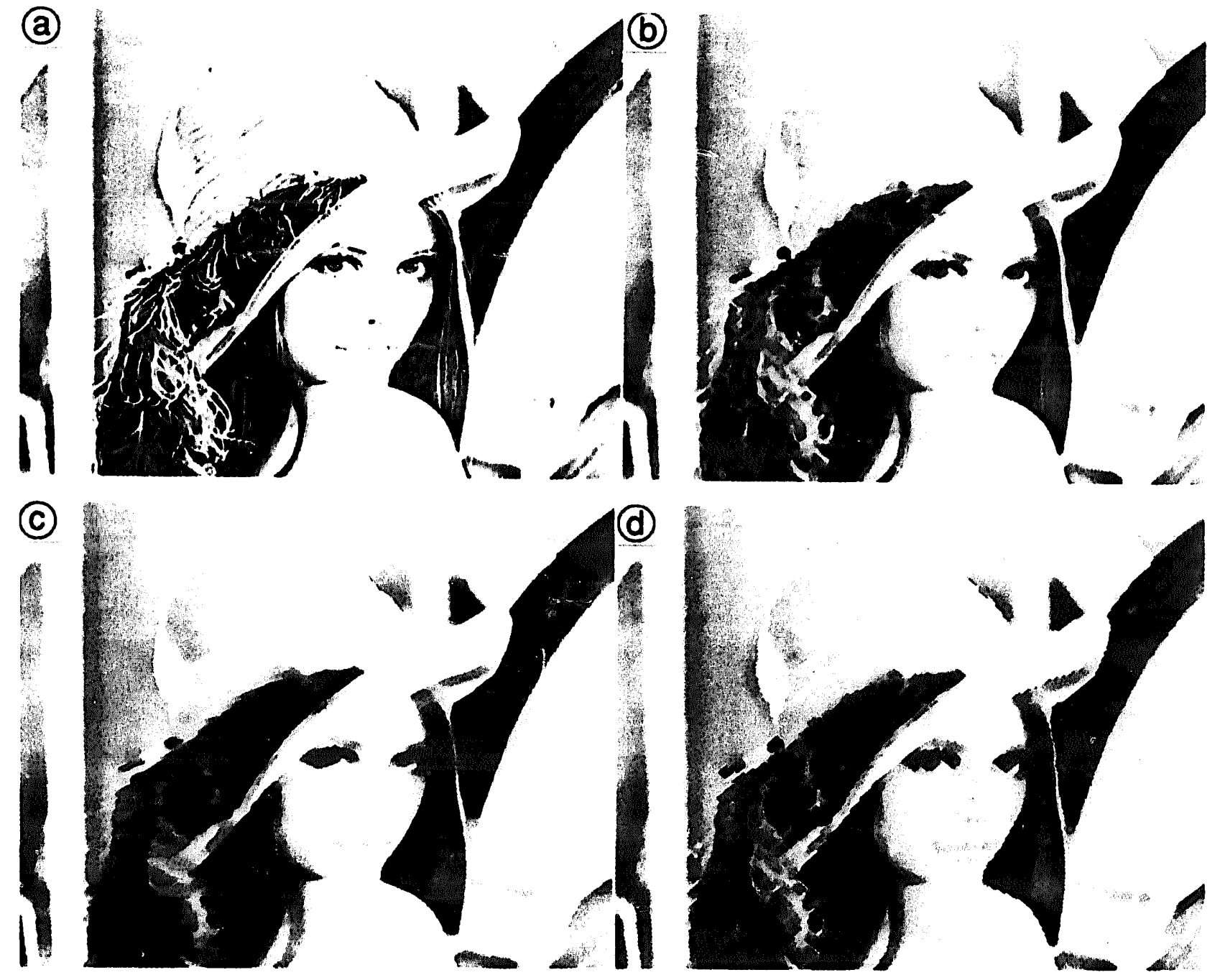

Fig. 12. (a) Original test picture - Lena; (b) result of a down and up conversion for $L=K=1$ and with $3 \times 3$ morphological decimation and interpolation filters (SNR $=24.24 \mathrm{SB}$ ); (c) result of a down and up conversion for $L=1, K=2$ and with $5 \times 5$ morphological decination and interpolation filters $(S N R=19.99 \mathrm{~dB})$; (d) result of a down and up conversion for $L=2, K=2$ and with $3 \times 3$ morphological decimation and interpolation filters $(S N R=20.14 \mathrm{~dB})$.

Fig. 12(a). The output SNR of the experimental result illustrated in Fig. 12(b) is $24.24 \mathrm{~dB}$. The result corresponding to $L=K=1$ preserves the maximal horizontal and vertical resolution as expected.

With reference to the second experiment with the parameters $L=1, K=2$, the morphological decimation and interpolation filters, with $5 \times 5$ structuring elements, are used. The output picture is given in Fig. 12(c) of which SNR is $19.99 \mathrm{~dB}$. Since the vertical sampling ra'ce and resolution are: decimated by a factor of 4 , the bluring effect becomes visible. However, there is no perceptible dis- tortion in the low-frequency areas of the output image. The results for the parameters $L=K=2$ and with $3 \times 3$ morphological filters are shown in Fig. 12(d) of which SNR is $20.14 \mathrm{~dB}$. In general, the smaller the structuring element of the morphological filter is. the higher the horizontal/vertical resolution of the result is.

For carrying out the test of conversion from an $\left(L_{1}, K_{1}\right)$ to an $\left(L_{2}, K_{2}\right)$ quincunx structure, the picture 'LENA' is used, and the system diagram has been presented in Fig. 8. The output results corresponding to the conversion for $\left(L_{1}=1, K_{1}=1\right.$ to 


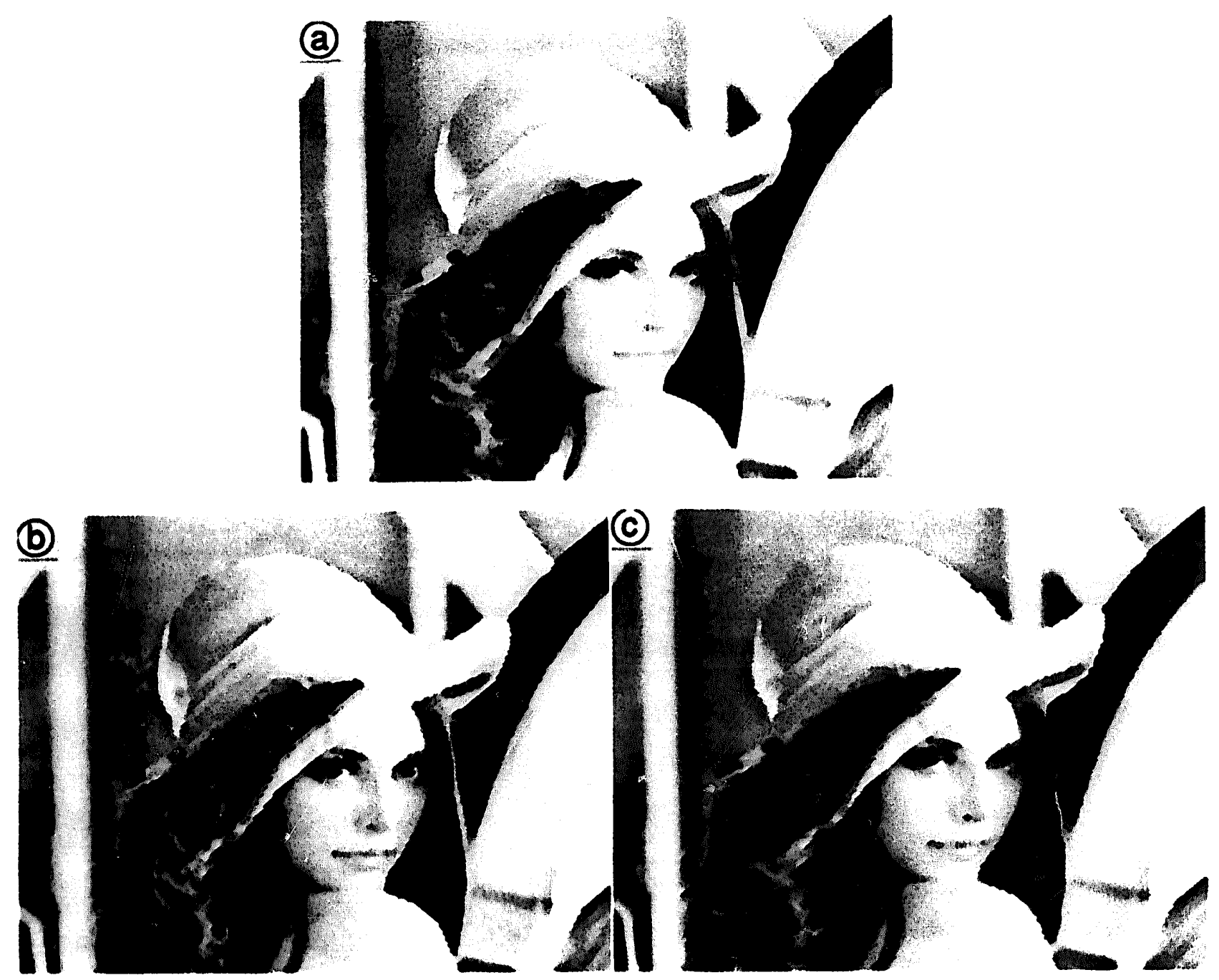

Fig. 13. Conversion from the $\left(L_{1}, K_{1}\right)$ quincunx structure to the $\left(L_{2}, K_{2}\right)$ quincunx structure by morphological filters; $\left(L_{2}, K_{2}\right)$ quincunx structure output $y(m, n)$, but filled with nearest neighbor pixel values: (a) from $\left(L_{1}=1, K_{1}=1\right)$ to $\left(L_{2}=1, K_{2}=2\right)$; (b) from $\left(L_{1}=1\right.$, $\left.K_{1}=1\right)$ to $\left(L_{2}=2, K_{2}=2\right)$; (c) from $\left(L_{1}=1, K_{1}=2\right)$ to $\left(L_{2}=2, K_{2}=2\right)$.

$\left.L_{2}=1, K_{2}=2\right),\left(L_{1}=1, K_{1}=1\right.$ to $L_{2}=2$, $\left.K_{2}=2\right)$ and $\left(L_{1}=1, K_{1}=2\right.$ to $\left.L_{2}=2, K_{2}=2\right)$ are shown in Figs. 13(a)-13(c), respectively. In order to view the final quincunx structure output images more clearly, the decimated/skipped pixels in $\left(L_{2}, K_{2}\right)$ subsampling have been filled with the gray level of the nearest neighbor nondecimated pixel.

As to the conversion from an $\left(L_{1}=1, K_{1}=2\right)$ rectangular sampling structure to an $\left(L_{2}=1\right.$, $K_{2}=2$ ) quincunx sampling structure, the test picture 'LENA' is also used, and the system diagram has been presented in Fig. 10. Fig. 14 shows the quincunx structure output $y(m, n)$ filled with the nearest neighbor pixel values.

From the experimental results, we observe that, in the output images, the edges are preserved; details appear more flat and fine details less than the size of the structuring elements will be suppressed by morphology filtering. The picture quality shown in Fig. 15(b) $(\mathrm{SNR}=24.12 \mathrm{~dB})$ seems satisfactory and is compatible to the linear-filtering approach shown in Fig. 15(c) (SNR = 27.15 dB), which is processed by a linear filter realized by the convolution 


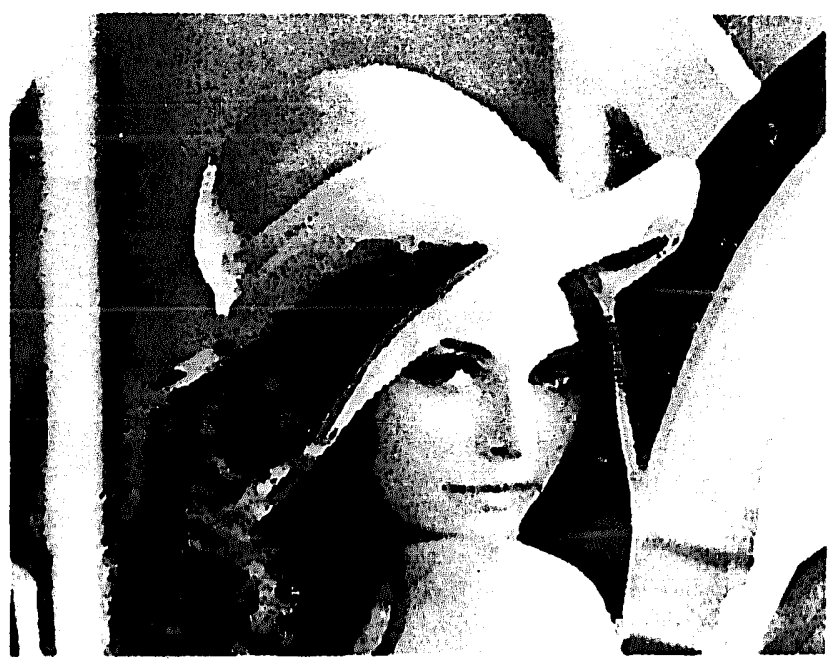

Fig. 14. Conversion from the $\left(L_{1}=1, K_{1}=2\right)$ rectangular structure to the $\left(L_{2}=1, K_{2}=2\right)$ quincunx structure by morphological filters: $\left(L_{2}, K_{2}\right)$ quincunx structure output $y(m, n)$, but filled with nearest neighbor pixel values.
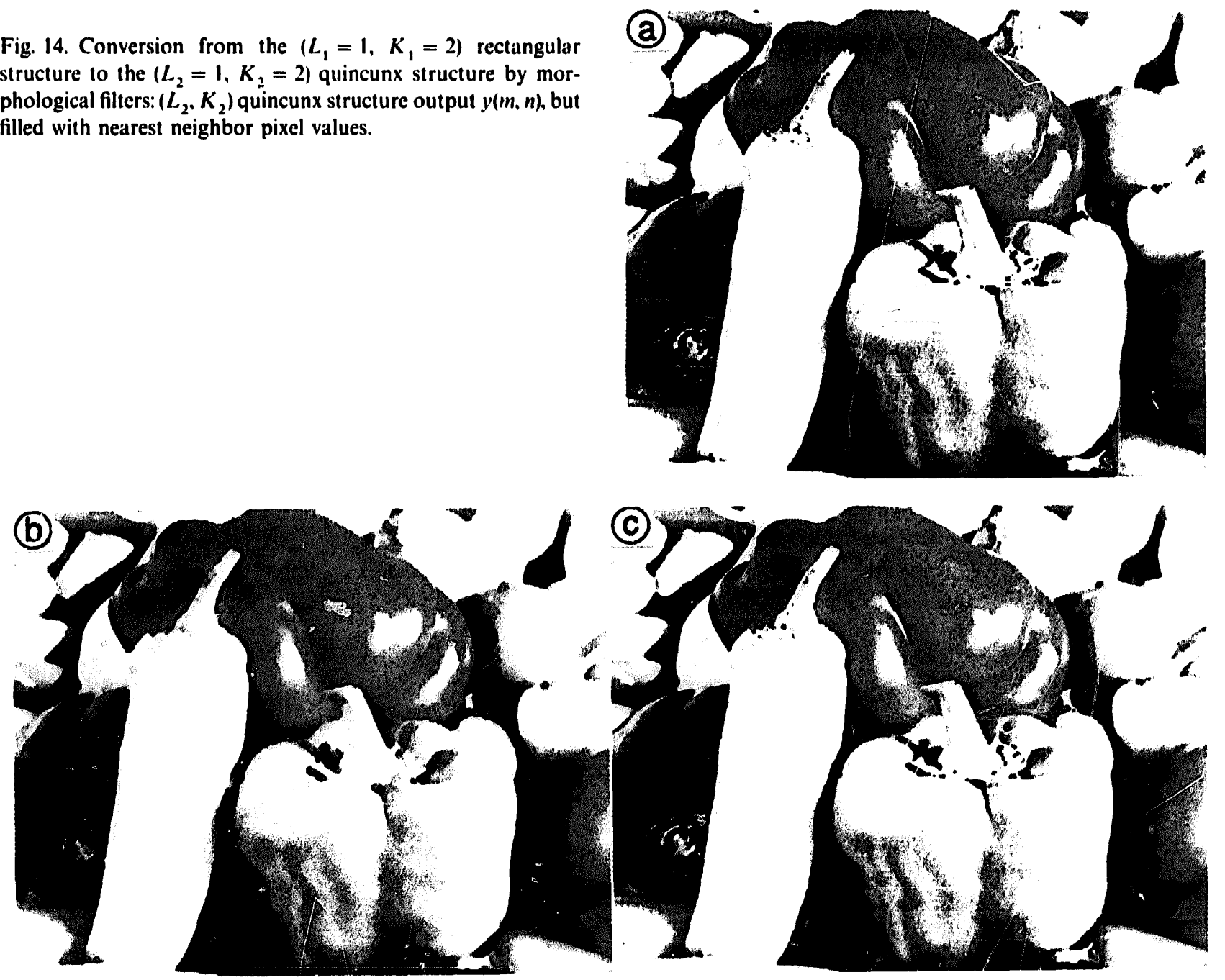

Fig. 15. (a) Original test picture - Vegetable; (b) result of a down and up conversion for $L=K=1$ and with $3 \times 3$ morphological decimation and interpolation filters $(S N R=24.12 \mathrm{~dB})$; (c) result of a down and up conversion for $L=K=1$ and with $3 \times 3$ linear decimation and interpolation filters $(\mathrm{SNR}=27.15 \mathrm{~dB})$. 
with $3 \times 3$ window for the sampling structure down and up conversions at $L=K=1$.

\section{Conclusions}

An effective sampling structure conversion using mathematical morphology has been presented in this paper. 2-D diamond-shaped morphological decimation and interpolation filters are constructed to allow a conversion from orthogonal to quincunx or quincunx to quincunx structure very efficiently. The morphological filters are relatively simple and inherently parallel. They are also suitable for very fast hardware architectures and VLSI implementation. Some experimental results have shown the effectiveness of this approach.

\section{References}

[1] R.M. Haralick and X. Zhuang, "Pipeline architectures for morphological image analysis", Machine Vision and Application, Vol. 1, 1988, pp. 23-40.

[2] R.M. Haralick. S.R. Sternberg and X. Zhuang. "Image analysis using mathematical morphology", IEEE Trans. Pattern Anal. Machine Intell., Vol. PAMI-9, No. 4, 1987, pp. $532-550$

[3] A. Knoll. "Filter design for the interpolation of highly subsampled pictures". Signal Processing: Image Commmmication. Vol. 3, Nos. 2 3, June 1991, pp. 239-248.

[4] G. Matheron, Random Sets and Integral Geometry. Wiley. New York, 1975.

[5] J. Serra, Image Analysis and Mathematical Morpholog̣!. Academic Press, Londnn, 1982.

[6] P. Siohan, "2-D FIR filter design for sampling structure conversion". IEEE Trans. Circuits and Systems for Video Technology, Vol. 1, No. 4, December 1991, pp. $337-350$. 